\title{
Hypercyclic Behavior of Translation Operators on Spaces of Analytic Functions on Hilbert Spaces
}

\author{
Zoryana Mozhyrovska ${ }^{1}$ and Andriy V. Zagorodnyuk ${ }^{2}$ \\ ${ }^{1}$ Lviv Commercial Academy, 10 Tugan-Baranovsky Street, Lviv 79005, Ukraine \\ ${ }^{2}$ Vasyl Stefanyk Precarpathian National University, 57 Shevchenko Street, Ivano-Frankivsk 76018, Ukraine \\ Correspondence should be addressed to Andriy V. Zagorodnyuk; andriyzag@yahoo.com
}

Received 17 March 2015; Accepted 19 May 2015

Academic Editor: Eva A. Gallardo Gutiérrez

Copyright (c) 2015 Z. Mozhyrovska and A. V. Zagorodnyuk. This is an open access article distributed under the Creative Commons Attribution License, which permits unrestricted use, distribution, and reproduction in any medium, provided the original work is properly cited.

We consider special Hilbert spaces of analytic functions of many infinite variables and examine composition operators on these spaces. In particular, we prove that under some conditions a translation operator is bounded and hypercyclic.

\section{Introduction}

Let $X$ be a Fréchet linear space. An operator $T: X \rightarrow X$ is called hypercyclic if there is a vector $x \in X$ whose orbit under $T$,

$$
\operatorname{Orb}(T, x)=\left\{x, T x, T^{2} x, \ldots\right\},
$$

is dense in $X$. Every such vector $x$ is called hypercyclic for $T$. It is well known that a hypercyclic operator can exist only in separable infinite-dimensional spaces (see [1]).

As for first results related to hypercyclic operators there are classical works of Birkhoff [2] and MacLane [3] showing that the operators of translation and differentiation, acting on the space of entire functions of one complex variable, are hypercyclic. There are many results related to hypercyclic operators on spaces of analytic functions on finite and infinite-dimensional spaces (see, e.g., $[1,4,5]$ ). Motivated by these results, we examine the hypercyclic behavior of composition operators on Hilbert spaces of entire functions of many infinite variables.

Let us recall that an operator $C_{\Phi}$ on the space of entire functions on $\mathbb{C}^{n} ; H\left(\mathbb{C}^{n}\right)$ is said to be a composition operator if $C_{\Phi} f(x)=f(\Phi(x))$ for some analytic map $\Phi: \mathbb{C}^{n} \rightarrow$ $\mathbb{C}^{n}$. According to the Birkhoff result [2] the operator of composition with translation $x \mapsto x+a, a \neq 0$, and $T_{a}$ : $f(x) \mapsto f(x+a)$ is hypercyclic in the space of entire functions $H(\mathbb{C})$ on the complex plane $\mathbb{C}$. Godefroy and Shapiro in [6] generalized this result for the translation operator on $H\left(\mathbb{C}^{n}\right)$, endowed with the topology of uniform convergence on compact subsets. Aron and Bès in [7] proved that the operator of composition with translation $T_{a}$ is hypercyclic in the space of weakly continuous analytic functions on all bounded subsets of a separable Banach space $X$ which are bounded on bounded subsets. Hypercyclic composition operators on spaces of analytic functions of finite and infinite many variables were studied also in [8]. In [9] Chan and Shapiro show that $T_{a}$ is hypercyclic in various Hilbert spaces of entire functions on $\mathbb{C}$. More detailed, they considered Hilbert spaces of entire functions of one complex variable $f(z)=\sum_{n=1}^{\infty} f_{n} z^{n}$ with norms $\|f\|_{2, \gamma}^{2}=\sum_{n=1}^{\infty} \gamma_{n}^{-2}\left|f_{n}\right|^{2}$ for appropriated sequence of positive numbers and showed that if $n \gamma_{n} / \gamma_{n-1}$ is monotonically decreasing, then $T_{a}$ is hypercyclic.

The purpose of this paper is to prove a generalization of the Chan and Shapiro's result for Hilbert spaces of entire functions of infinite many variables. In order to do it we consider in Section 1 a general construction of analytic functions on a Hilbert space which is related to generalized Fock space. In Section 2 we study special cases of Hilbert spaces of entire functions on a separable Hilbert space. In Section 3 we establish some conditions under which the translation operator is bounded and hypercyclic on these special spaces. 
There is a general sufficient condition for hypercyclicity. This condition is inspired in the so-called Hypercyclicity Criterion given by Kitai [10] in her unpublished Ph.D. thesis and rediscovered by Gethner and Shapiro [11]. We use the general form of this Criterion as given in [7]. It may be stated as follows.

Theorem 1 (Hypercyclicity Criterion). Let $X$ be separable complete linear metric space and let $T: X \rightarrow X$ be linear continuous operator. Suppose there exist $X_{0}, Y_{0}$ of $X, a$ sequence $\left(n_{k}\right)$ of positive integers, and a sequence of mappings (possibly nonlinear, possibly not continuous) $S_{n_{k}}: Y_{0} \rightarrow X$ so that

(i) $T^{n_{k}} \rightarrow 0$ for $k \rightarrow \infty$ pointwise on $X_{0}$,

(ii) $S_{n_{k}} \rightarrow 0$ for $k \rightarrow \infty$ pointwise on $Y_{0}$,

(iii) $T^{n_{k}} S_{n_{k}}=I$ on $Y_{0}$ (I is identity operator);

then $T$ is hypercyclic.

For background on analytic functions on Banach spaces we refer the reader to $[12,13]$.

\section{Symmetric Fock Spaces and Analytic Functions}

Let $E$ be a complex separable Hilbert space with an orthonormal basis $\left(e_{i}\right)_{i=1}^{\infty}$ endowed with the scalar product $(x \mid y)_{E}$ and the norm $\|x\|_{E}=(x \mid x)_{E}^{1 / 2}, x, y \in E$. Clearly, for every $n \in \mathbb{N}$ the $n$th tensor power $\otimes^{n} E$ is defined to be complex linear span of elements

$$
\left\{x_{1} \otimes \cdots \otimes x_{n}: x_{1}, \ldots, x_{n} \in E\right\} .
$$

It is well-known that it is possible to define a norm $\|\cdot\|_{\otimes_{h}^{n} E}$ on the vector space $\otimes^{n} E$ such that the corresponding completion $\otimes_{h}^{n} E$ is a Hilbert space. More exactly, the scalar product on $\otimes_{h}^{n} E$ is defined by the equality

$$
\begin{gathered}
\left\langle x_{1} \otimes \cdots \otimes x_{n} \mid y_{1} \otimes \cdots \otimes y_{n}\right\rangle_{\otimes_{h}^{n} E} \\
=\left(x_{1} \mid y_{1}\right)_{E} \cdots\left(x_{n} \mid y_{n}\right)_{E}
\end{gathered}
$$

for all $x_{i}, y_{i} \in E, i=1, \ldots, n$. Let $[i]$ denote a multi-index $\left(i_{1}, \ldots, i_{n}\right) \in \mathbb{N}^{n}$. Since the system

$$
\left\{e_{i_{1}} \otimes \cdots \otimes e_{i_{n}} \in \otimes^{n} E:[i] \in \mathbb{N}^{n}\right\}
$$

forms an orthonormal basis in $\otimes_{h}^{n} E$, every such vector $w \epsilon$ $\otimes_{h}^{n} E$ can be represented by the Fourier series expansion

$$
\omega=\sum_{[i] \in \mathbb{N}^{n}} \alpha_{[i]} e_{i_{1}} \otimes \cdots \otimes e_{i_{n}},
$$

and we put

$$
\|\omega\|_{\otimes_{h}^{n} E}=\langle\omega \mid \omega\rangle_{\bigotimes_{h}^{n} E}^{1 / 2}=\left(\sum_{[i] \in \mathbb{N}^{n}}\left|\alpha_{[i]}\right|^{2}\right)^{1 / 2} .
$$

It is clear that the above norm, generated by the scalar product, is a cross-norm on $\otimes_{h}^{n} E$; that is,

$$
\left\|x_{1} \otimes \cdots \otimes x_{n}\right\|_{\otimes_{h}^{n} E}=\left\|x_{1}\right\|_{E} \cdots\left\|x_{n}\right\|_{E} .
$$

We denote by $\otimes_{s}^{n} E$ the $n$-fold symmetric algebraic tensor product of space $E$. Every element from $\otimes_{s}^{n} E$ can be defined by formula

$$
x_{1} \otimes_{s} \cdots \otimes_{s} x_{n}:=\frac{1}{n !} \sum_{\sigma \in S_{n}} x_{\sigma(1)} \otimes \cdots \otimes x_{\sigma(n)},
$$

where $x_{1}, \ldots, x_{n} \in E$ and $S_{n}$ is the group of permutations on the set $\{1, \ldots, n\}$.

We will use the following notations $e_{i}^{\otimes k}=\underbrace{e_{i} \otimes \cdots \otimes e_{i}}_{k}$ for any $k, i \in \mathbb{N}$. We denote by $(k)$ an arbitrary multi-index $\left(k_{1}, \ldots, k_{n}\right) \in \mathbb{Z}_{+}^{n},|(k)|=\sum_{i} k_{i}$, and $(k) !=\prod_{i} k_{i} !$. The vectors

$$
\begin{aligned}
& \left\{e_{[i]}^{\otimes(k)}:=e_{i_{1}}^{\otimes k_{1}} \otimes_{s} \cdots \otimes_{s} e_{i_{n}}^{\otimes k_{n}}:[i] \in \mathbb{N}^{n},(k) \in \mathbb{Z}_{+}^{n},|(k)|\right. \\
& \quad=n\}
\end{aligned}
$$

form an orthogonal basis in the closure $\otimes_{s, h}^{n} E$ of $\otimes_{s}^{n} E$ in $\otimes_{h}^{n} E$ and

$$
\left\|e_{[i]}^{\otimes(k)}\right\|_{\otimes_{h}^{n} E}=\sqrt{\frac{(k) !}{n !}}, \quad n=|(k)| .
$$

By Hermitian duality of a Hilbert space $E$ we can define the relation

$$
E^{*}=\left\{y^{*}:=\langle\cdot \mid y\rangle_{E}: y \in E\right\} .
$$

Note that the classical symmetric Fock space $\mathscr{F}$ is the Hilbert direct sum of $\otimes_{s, h}^{n} E, n=0,1, \ldots$, where $\otimes_{s, h}^{0} E=\mathbb{C}$. This space is predual to a space of analytic functions on the unit ball of $E$ [14].

We say that a Hilbert space $\mathscr{F}_{\eta}$ with an arbitrary Hilbert norm $\|\cdot\|_{\eta}$ is (generalized) symmetric Fock space over a given Hilbert space $E$ if vectors $1, e_{[i]}^{(k)}=e_{i_{1}}^{\otimes k_{1}} \otimes_{s} \cdots \otimes_{s} e_{i_{n}}^{\otimes k_{n}},(k \in \mathbb{N}$, $k_{1}+\cdots+k_{n}=n, i_{1}<\cdots<i_{n}$ ) form an orthogonal basis in $\mathscr{F}_{\eta}$. Thus $\mathscr{F}_{\eta}$ can be represented by the Hilbert direct sum of symmetric tensor powers:

$$
\mathscr{F}_{\eta}=\mathbb{C} \oplus E \oplus \otimes_{s}^{2} E \oplus \cdots \oplus \otimes_{s}^{n} E \oplus \cdots .
$$

Evidently, the norm $\|\cdot\|_{\eta}$ is completely defined by its value on the basis vectors. Hence, setting $\left\|e_{[i]}^{\otimes(k)}\right\|_{\eta}$ by arbitrary positive numbers, we can get various symmetric Fock spaces over $E$. Let $\langle\cdot \mid \cdot\rangle_{\eta}$ be the scalar product in $\mathscr{F}_{\eta}$. series

Put $c_{[i]}^{(k)}:=\left\|e_{[i]}^{(k)}\right\|_{\eta}^{-2}$ and $c_{0}=1$. Let us consider a power

$$
\eta(x)=\sum_{k_{1}+\cdots+k_{n}=0}^{\infty} \sum_{i_{1}<\cdots<i_{n}} c_{i_{1} \cdots i_{n}}^{k_{1} \cdots k_{n}} x_{i_{1}}^{k_{1}} \cdots x_{i_{n}}^{k_{n}} e_{i_{1}}^{\otimes k_{1}} \cdots e_{i_{n}}^{\otimes k_{n}}
$$

$$
=\sum_{|(k)|=0}^{\infty} \sum_{[i] \in \mathbb{N}^{n}} c_{[i]}^{(k)} x_{[i]}^{(k)} e_{[i]}^{\otimes(k)}
$$

for any $x=\sum_{i=1}^{\infty} x_{i} e_{i} \in E$. 
Theorem 2. Suppose that there are constants $c>0$ and $M>0$ such that for all multi-indexes $[i] \in \mathbb{N}^{n},(k) \in \mathbb{Z}_{+}^{n}$, and $n=$ $k_{1}+\cdots+k_{n}$ inequalities

$$
\begin{aligned}
0 & <c_{[i]}^{(k)}=c_{i_{1} \cdots i_{n}}^{k_{1} \cdots k_{n}} \leq c M^{2 n} \frac{\left(k_{1}+\cdots+k_{n}\right) !}{k_{1} ! \cdots k_{n} !} \\
& =c M^{2 n} \frac{n !}{k_{1} ! \cdots k_{n} !}
\end{aligned}
$$

hold. Then there exists an open subset $U \subset E, U \ni 0$ such that

(i) the series (13) is convergent for every $x \in U$ and $\eta$ is an analytic map from $U$ into $\mathscr{F}_{\eta}$,

(ii) for every $\varphi \in \mathscr{F}_{\eta}$ the map $f_{\varphi}(x)=\langle\eta(x) \mid \varphi\rangle_{\eta}$ is an analytic function on $U$,

(iii) the function $\left\langle\eta(x) \mid e_{[i]}^{\otimes(k)}\right\rangle_{\eta}$ is an n-homogeneous polynomial and

$$
\left\langle\eta(x) \mid e_{[i]}^{\otimes(k)}\right\rangle_{\eta}=x_{i_{1}}^{k_{1}} \cdots x_{i_{n}}^{k_{n}} .
$$

We can find the proof in [15, Proposition 4.22].

Let us denote by $\mathscr{H}_{\eta}$ the Hilbert space of analytic function $f_{\phi}=\langle\eta(\cdot) \mid \phi\rangle_{\eta}$ that is Hermitian duality to $\mathscr{F}_{\eta}$. We will use the same symbol $\langle\cdot \mid \cdot\rangle_{\eta}$ for the scalar product in $\mathscr{H}_{\eta}$.

For any vector $f \in \mathscr{H}_{\eta}$ denote $\bar{f} \in \mathscr{F}_{\eta}$ such that $f=\langle\cdot|$ $\bar{f}\rangle_{\eta}$. In particular, $f(x)=\langle\eta(x) \mid \bar{f}\rangle_{\eta}$. Also by $\bar{g}, g \in \mathscr{F}_{\eta}$ we mean a vector from $\mathscr{H}_{\eta}$ such that $g=\langle\cdot \mid \bar{g}\rangle_{\eta}$.

We recall definition of reproducing kernel.

Definition 3. Let $Z$ be an abstract set and let $\mathscr{H}$ be an Hilbert space of complex valued functions $f$ on $Z$ with the scalar product $\langle\cdot \mid \cdot\rangle_{\mathscr{H}}$. A function $K(x, z)$ defined on $Z \times Z$ is called reproducing kernel of closed subspace $\mathscr{H}_{K} \subset \mathscr{H}$ if

(i) for any fixed $z \in Z$, the kernel $K(x, z)$ belongs to $\mathscr{H}_{K}$ as a function of $x \in Z$;

(ii) for any $f \in \mathscr{H}_{K}$ and for any $z \in Z$

$$
f(z)=\langle f(\cdot) \mid K(\cdot, z)\rangle_{\mathscr{H}} .
$$

Hilbert space $\mathscr{H}_{K}$ is called space with reproducing kernel or functional Hilbert space.

Let $h: Z \rightarrow \mathscr{H}$ be a function on $Z$ such that for every $f \in \mathscr{H}_{K}$ and $x \in Z$

$$
f(x)=\langle f(\cdot) \mid h(x)\rangle_{\mathscr{H}} .
$$

Theorem 4. The function $K(x, z)=\langle h(z) \mid h(x)\rangle_{\mathscr{H}}$ is reproducing kernel for $\mathscr{H}_{K}$.

We may see the proof in $[16$, p. 21$]$.

Proposition 5. A map $K: E \times E \rightarrow \mathbb{C}$ defined by

$$
K(x, z)=\langle\bar{\eta}(x) \mid \bar{\eta}(z)\rangle_{\eta}=\langle\eta(z) \mid \eta(x)\rangle_{\eta}
$$

is a reproducing kernel for $\mathscr{H}_{\eta}$.
The proof immediately follows from Theorem 4 for $h(x):=\bar{\eta}(x)$.

Since $\eta$ generates the reproducing kernel of $\mathscr{H}_{\eta}$ we say that $\eta$ is a reproducing function of $\mathscr{H}_{\eta}$.

Example 6. For an arbitrary positive integer $m$ set

$$
\begin{aligned}
\eta^{(m)} & (x) \\
& =\sum_{k=0}^{\infty} \frac{(m-1+k) !}{(m-1) ! k !} x^{k} \\
& =\sum_{k=0}^{\infty} \frac{(m-1+k) !}{(m-1) ! k !}\left(\sum_{i=1}^{\infty} x_{i} e_{i}\right)^{k} \\
& =\sum_{|(k)|=0}^{\infty} \sum_{[i] \in \mathbb{N}^{n}} \frac{\left(k_{1}+\cdots+k_{n}+m-1\right) !}{(m-1) ! k_{1} ! \cdots k_{n} !} e_{[i]}^{\otimes(k)} x_{[i]}^{(k)},
\end{aligned}
$$

where $(k)=\left(k_{1}, \ldots, k_{n}\right), n \in \mathbb{Z}_{+}$. Thus

$$
c_{[i]}^{(k)}=\frac{\left(k_{1}+\cdots+k_{n}+m-1\right) !}{(m-1) ! k_{1} ! \cdots k_{n} !} .
$$

We denote by $B:=\left\{x \in E:\|x\|_{E}<1\right\}$ unit ball on $E$. It is easy to see that $\eta^{(m)}$ is an analytic map from the unit ball $B \subset E$ to $\mathscr{F}_{\eta^{(m)}}$ for every $m$ and

$$
\begin{aligned}
\left\|\eta^{(m)}(x)\right\|_{\eta} & =\left(\sum_{k=0}^{\infty} \frac{(m-1+k) !}{(m-1) ! k !}\|x\|_{E}^{2 k}\right)^{1 / 2} \\
& =\left(\frac{1}{\left(1-\|x\|_{E}^{2}\right)^{m}}\right)^{1 / 2} .
\end{aligned}
$$

If $m=1$ and $X=\mathbb{C}^{n}$, then this space is called DruryArveson Hardy space [17]. As well the space coincides with Besov-Sobolev space $B_{2}^{1 / 2}$ of analytic functions on open unit ball in $\mathbb{C}^{n}$. Note that $\mathscr{H}_{\eta^{(m)}}$ coincides with the classical Hardy space on the unit ball if (and only if) $\operatorname{dim} E=m$.

Note that various Hilbert spaces of analytic functions of infinite many variables are studied in [18-22].

\section{Hilbert Spaces of Entire Functions}

In this section we consider the case when $\mathscr{H}_{\eta}=\mathscr{F}_{\eta}^{*}$ consists with entire functions on $E$.

Proposition 7. Suppose that there exists a constant $c>0$ and a sequence of positive numbers $\left(M_{n}\right), M_{n} \rightarrow 0$ as $n \rightarrow \infty$, such that

$$
c_{[i]}^{(k)} \leq c M_{n}^{2 n} \frac{n !}{k_{1} ! \cdots k_{n} !},
$$


where $c_{[i]}^{(k)}=\left\|e_{[i]}^{\otimes(k)}\right\|_{\eta}^{-2}$ and $e_{[i]}^{\otimes(k)}$ is an orthogonal basis in $\mathscr{F}_{\eta}$. Then $\mathscr{H}_{\eta}=\mathscr{F}_{\eta}^{*}$ is a Hilbert space of entire functions of bounded type that is bounded on bounded subsets on $E$.

The proof is in [15, Proposition 4.25].

The next proposition gives another test for $\mathscr{H}_{\eta}$ to be a space of entire functions.

Proposition 8. Suppose that $\left\|\eta_{n+1}\right\|_{\eta} /\left\|\eta_{n}\right\|_{\eta}$ decreases to zero as $n$ increases to infinity. Then $\mathscr{H}_{\eta}$ consists with bounded-type entire functions, where

$$
\begin{aligned}
& \eta_{n}(x)=\sum_{|(k)|=n[i] \in \mathbb{N}^{n}} \sum_{[i]} x_{[i]}^{(k)} x_{[i]}^{(k)} e_{[(k)}^{\otimes(k)} \\
& \left\|\eta_{n}\right\|_{\eta}=\sup _{\|x\| \leq 1}\left\|\eta_{n}(x)\right\|_{\eta} .
\end{aligned}
$$

Proof. By the ratio test the power series $\sum_{n=0}^{\infty}\left\|\eta_{n}\right\|_{\eta} t^{n}$ is absolutely convergent for every $t \in \mathbb{C}$. Thus, by the CauchyHadamard formula,

$$
\limsup _{n \rightarrow \infty}\left(\left\|\eta_{n}\right\|_{\eta}^{1 / n}\right)^{-1}=\infty
$$

and $\eta$ is hence an entire mapping.

Example 9. Let

$$
\eta(x)=\sum_{n=0}^{\infty} \frac{x^{\otimes n}}{n !}
$$

where $x \in E$. Denote by $H^{2}(E)$ the corresponding space $\mathscr{H}_{\eta}$. It is easy to see that $H^{2}(E)$ consists of bounded-type entire functions on $E$ and

$$
\left\|e_{[i]}^{\otimes(k)}\right\|_{\eta}^{2}=k_{1} ! \cdots k_{n} !
$$

The reproducing kernel of this space is

$$
\begin{aligned}
& k(z, x)=\langle\eta(x) \mid \eta(z)\rangle_{\eta}=\sum_{n=0}^{\infty} \frac{\left\langle x^{\otimes n} \mid z^{\otimes n}\right\rangle_{\eta}}{(n !)^{2}} \\
& =\sum_{n=0}^{\infty} \sum_{(k) \in \mathbb{Z}_{+}^{n}[i] \in \mathbb{N}^{n}} \frac{1}{(n !)^{2}}\left(\frac{n !}{k_{1} ! \cdots k_{n} !}\right)^{2} \\
& \cdot\left\|e_{i_{1}}^{\otimes k_{1}} \cdots e_{i_{n}}^{\otimes k_{n}}\right\|_{\eta}^{2} x_{i_{1}}^{k_{1}} \cdots x_{i_{n}}^{k_{n}} z_{i_{1}}^{k_{1}} \cdots z_{i_{n}}^{k_{n}} \\
& =\sum_{n=0}^{\infty} \sum_{(k) \in \mathbb{Z}_{+}^{n}[i] \in \mathbb{N}^{n}} \sum_{k_{1} ! \cdots k_{n} !} x_{[i]}^{(k)} z_{[i]}^{(k)}=\sum_{n=0}^{\infty} \frac{(x \mid z)_{E}^{n}}{n !} \\
& =e^{(x \mid z)_{E}}
\end{aligned}
$$

and for every function from $H^{2}(E)$ there exists $w \in \mathscr{F}_{\eta}$ such that

$$
f_{w}(x)=\langle\eta(x) \mid w\rangle_{\eta}
$$

for any vector $w \in \mathscr{F}_{\eta}$. According to [22] $H^{2}(E)$ is an infinite tensor product of

$$
H^{2}(\mathbb{C})=\left\{f \in H(\mathbb{C}): \int_{\mathbb{C}}|f(z)|^{2} e^{-|z|^{2}} d z<\infty\right\},
$$

where $d z$ is the Lebesgue measure on $\mathbb{C}$.

Let $D$ be the open unit disk in $\mathbb{C}$. Denote by $\Gamma_{r}, 0<r \leq \infty$, the set of all analytic functions on $r D$ if $r<\infty$ and on $\mathbb{C}$ if $r=\infty, \gamma(t)=\sum_{k=0}^{\infty} \gamma_{k} t^{k}$ such that $\gamma_{k}>0, k \in \mathbb{Z}_{+}$. Let $\Omega$ be a stand for both $r D$ and $\mathbb{C}$. Evidently, $\Gamma_{r}$ is an open convex subset of the Fréchet space of all analytic functions $H(\Omega)$ on $\Omega$.

Proposition 10. For a given $\gamma(t)=\sum_{k=0}^{\infty} \gamma_{k} t^{k} \in \Gamma_{r}$ and $a$ Hilbert space E the function

$$
\eta^{(\gamma)}(x)=\sum_{k=0}^{\infty} \gamma_{k} x^{k}
$$

is analytic on the ball $r B \subset E$ (where $r B=E$ if $r=\infty)$ to $\mathscr{F}_{\eta^{(\gamma)}}$ and

$$
\left\|\eta^{(\gamma)}(x)\right\|_{\eta}^{2}=\left\langle\eta^{(\gamma)}(x) \mid \eta^{(\gamma)}(x)\right\rangle_{\eta}=\gamma\left(\|x\|_{E}^{2}\right)
$$

for every $x \in r B$.

The proof is in [15, Proposition 4.28].

We say that $\eta^{(\gamma)}$ is generated by $\gamma$. Note that the reproducing function $\eta^{(m)}$ in Example 6 is generated by $\gamma(t)=$ $1 /(1-t)^{m}$ and the reproducing function $\eta$ in Example 9 is generated by $\gamma(t)=e^{t}$.

Corollary 11. Let $\gamma(t)=\sum_{k=0}^{\infty} \gamma_{k} t^{k}, \gamma_{k}>0$, be an entire function of one complex variable such that $\gamma_{n+1} / \gamma_{n}$ decreases to zero as $n$ increases to $\infty$. Then $\eta^{(\gamma)}$ is a reproducing function of a Hilbert space $\mathscr{H}_{\eta^{(\gamma)}}$ of entire functions on the Hilbert space E.

Let $g(t)=\sum_{n=0}^{\infty} g_{n} t^{n}$ be an entire function of one complex variable. We are interested to know the following: Under which conditions does $g \circ \varphi(x)=g(\varphi(x))$ belong to $\mathscr{H}_{\eta}$ for a given $\varphi \in E^{*}$ ? Let $\varphi=\sum_{n=1}^{\infty} \varphi_{n}\left(\cdot \mid e_{n}\right)_{E}$. It is easy to see that if $w=\sum_{n=0}^{\infty} w_{n} \in \mathscr{F}_{\eta}$ where $w_{0}=\bar{g}_{0}$ and

$$
w_{n}=g_{n} \sum_{i=1}^{\infty}\left(\varphi_{i} e_{i}\right)^{\otimes n}=g_{n} \sum_{|(k)|=n[i] \in \mathbb{N}^{n}} \frac{n !}{(k) !} \varphi_{[i]}^{(k)} e_{[i]}^{\otimes(k)}
$$

then $g \circ \varphi(x)=\langle\eta(x) \mid w\rangle_{\eta}$. So

$$
\begin{aligned}
\|w\|_{\eta}^{2} & =\sum_{n=0}^{\infty}\left|g_{n}\right|^{2} \sum_{|(k)|=n[i] \in \mathbb{N}^{n}} \frac{n !}{(k) !}\left|\varphi_{[i]}^{(k)}\right|^{2}\left\|e_{[i]}^{\otimes(k)}\right\|^{2} \\
& =\sum_{n=0}^{\infty}\left|g_{n}\right|^{2} \sum_{|(k)|=n[i] \in \mathbb{N}^{n}} \frac{n !\left|\varphi_{[i]}^{(k)}\right|^{2}}{(k) ! c_{[i]}^{(k)}} .
\end{aligned}
$$


If $\eta$ is generated by an analytic function $\gamma(t)$ in the means of (30), then (33) can be rewritten by

$$
\|w\|_{\eta}^{2}=\sum_{n=0}^{\infty} \frac{\left|g_{n}\right|^{2}}{\gamma_{n}}\|\varphi\|^{2 n} .
$$

So we have proved the following proposition.

Proposition 12. Let $g(t)=\sum_{n=0}^{\infty} g_{n} t^{n}$ be an entire function of one complex variable. Then $g \circ \varphi(x)=g(\varphi(x))$ belongs to $\mathscr{H}_{\eta}$ for a given $\varphi \in E^{*}$ if and only if

$$
\sum_{n=0}^{\infty}\left|g_{n}\right|^{2} \sum_{|(k)|=n} \sum_{[i] \in \mathbb{N}^{n}} \frac{n !\left|\varphi_{[i]}^{(k)}\right|^{2}}{(k) ! c_{[i]}^{(k)}}<\infty .
$$

And if $\eta$ is generated by an analytic function $\gamma(t)$ in the means of (30), then the condition may be written by

$$
\sum_{n=0}^{\infty} \frac{\left|g_{n}\right|^{2}}{\gamma_{n}}\|\varphi\|^{2 n}<\infty
$$

In the case when $g(t)=e^{t}$ we can write

$$
\begin{gathered}
\sum_{n=0}^{\infty} \sum_{|(k)|=n} \sum_{[i] \in \mathbb{N}^{n}} \frac{\left|\varphi_{[i]}^{(k)}\right|^{2}}{n !(k) ! c_{[i]}^{(k)}}<\infty, \\
\sum_{n=0}^{\infty} \frac{\|\varphi\|^{2 n}}{(n !)^{2} \gamma_{n}}<\infty,
\end{gathered}
$$

respectively.

\section{Differentiation and Translation Operators on $\mathscr{H}_{\eta}$}

Let us consider a differentiation operator $D_{a}: \mathscr{H}_{\eta} \rightarrow \mathscr{H}_{\eta}$ :

$$
D_{a}(f(x))=\sum_{i=1}^{\infty} a_{i} \frac{\partial}{\partial x_{i}} f
$$

where $a=\left(a_{i}\right) \in \ell_{2}, x \in E$.

$D_{a}$ is well defined on an appropriated dense subspace in $\mathscr{H}_{\eta}$ containing linear functionals. It is clear that $D_{a}$ is defined on functions $e^{\varphi}, \varphi \in E^{*}$, if $e^{\varphi} \in \mathscr{H}_{\eta}$.

We will make use of the following two lemmas (cf. [7]).

Lemma 13. $\mathscr{B}=\left\{e^{\varphi}: \varphi \in E^{*}, e^{\varphi} \in \mathscr{H}_{\eta}\right\}$ is a linearly independent subset of $\mathscr{H}_{\eta}$.

Proof. Let $\left\{e^{\varphi_{i}}\right\}_{i \in I}$ be a maximal linearly independent subset of $\mathscr{B}$, where $I$ is a set of indexes. Fix $\varphi \in E^{*}$, and assume that there exist nonzero constants $c_{i_{1}}, \ldots, c_{i_{r}} \in \mathbb{C}$ so that

$$
c_{i_{1}} e^{\varphi_{i_{1}}}+\cdots+c_{i_{r}} e^{\varphi_{i_{r}}}=e^{\varphi} .
$$

Let $a \in E$ be arbitrary. Applying the differentiation operator $f \mapsto D_{a} f(\cdot)$ in (39), it follows that

$$
c_{i_{1}} \varphi_{i_{1}}(a) e^{\varphi_{i_{1}}}+\cdots+c_{i_{r}} \varphi_{i_{r}}(a) e^{\varphi_{i_{r}}}=\varphi(a) e^{\varphi} .
$$

Since $\left\{e^{\varphi_{i}}\right\}_{i \in I}$ is linearly independent and $c_{i_{1}}, \ldots, c_{i_{r}}$ are nonzero, by (39) and (40) we have

$$
\varphi_{i_{1}}(a)=\cdots=\varphi_{i_{r}}(a)=\varphi(a)
$$

Hence the set $\left\{\varphi_{i}\right\}_{i \in I}$ such that $\left\{e^{\varphi_{i}}\right\}_{i \in I}$ is maximal linearly independent subset which coincides with $E^{*}$ and so $\left\{e^{\varphi_{i}}\right\}_{i \in I}=$ $\mathscr{B}$.

Lemma 14. Let $U$ be a nonempty open subset of a ball in $E^{*}$ with radius $\delta$ and center in 0 . Suppose that $e^{\varphi} \in \mathscr{H}_{\eta}$ for every $\varphi \in U$. Then $S=\operatorname{span}\left\{e^{\varphi}: \varphi \in U\right\}$ is dense in $\mathscr{H}_{\eta}$

Proof. It is sufficient to establish that $\varphi^{n} \in \bar{S}$ for all $\varphi \in$ $U$ and $n \geq 1$. To test this assertion we use the method of mathematical induction. When $n=1$ the statement is obvious.

Suppose the claim is true for $n \leq k-1$. We prove this for $n=k$. Since $t \varphi \in U$, then for each $0<t<1$ we have

$$
g_{t}=\frac{1}{t^{k}}\left(e^{t \varphi}-1-t \varphi-\frac{[t \varphi]^{2}}{2 !}-\cdots-\frac{[t \varphi]^{k-1}}{(k-1) !}\right) \in \bar{S} \text {. }
$$

So given $x \in E$,

$$
\begin{aligned}
& \left|\left(g_{t}-\frac{\varphi^{k}}{k !}\right)(x)\right| \\
& =\left|\frac{1}{t^{k}}\left[e^{t \varphi}-1-t \varphi-\frac{[t \varphi]^{2}}{2 !}-\cdots-\frac{[t \varphi]^{k}}{k !}\right](x)\right| \\
& \leq\left|\frac{1}{t^{k}} \sum_{n \geq k+1} \frac{[t \varphi]^{n}}{n !}(x)\right| \leq t \sum_{n \geq k+1} t^{n-k-1} \frac{|\varphi(x)|^{n}}{n !} \\
& \leq t e^{\delta\|x\|_{E}} .
\end{aligned}
$$

Thus, $g_{t} \rightarrow \varphi^{k} / k$ ! for $t \rightarrow 0$ in $\mathscr{H}_{\eta}$ and $\varphi^{k} / k ! \in \bar{S}$. So the claim holds.

We will be interested in the operators of differentiation and translation on the Hilbert space $\mathscr{H}_{\eta}$.

Theorem 15. The operator $D_{a}$ is bounded on $\mathscr{H}_{\eta}$ if and only if the set $\left\{k_{j} \sqrt{c_{[i]}^{(k)}} / \sqrt{c_{[i]}^{\left(k^{j^{\prime}}\right)}}\right\}$ is bounded, where coefficients $c_{[i]}^{(k)}$ are defined in (13), $n \in \mathbb{N}$, $[i] \in \mathbb{N}^{n},(k) \in \mathbb{Z}_{+}^{n}, n=|(k)|$, and $\left(k^{j^{\prime}}\right)=\left(k_{1}, k_{2}, \ldots, k_{j}-1, \ldots, k_{n}\right)$.

Proof. The functions

$$
\begin{aligned}
\mathscr{E}_{[i]}^{(k)}(x) & =\frac{\left(x \mid e_{[i]}^{\otimes(k)}\right)}{\left\|e_{[i]}^{\otimes(k)}\right\|_{\eta}}=\left(x \mid e_{[i]}^{\otimes(k)}\right) \sqrt{c_{[i]}^{(k)}}=\sqrt{c_{[i]}^{(k)}} x_{[i]}^{(k)} \\
& =\sqrt{c_{i_{1}}^{k_{1}} \cdots c_{i_{n}}^{k_{n}}} x_{i_{1}}^{k_{1}} \cdots x_{i_{n}}^{k_{n}}
\end{aligned}
$$


form an orthonormal basis for $\mathscr{H}_{\eta}$. Define the 1th-derivative of $\mathscr{E}_{[i]}^{(k)}$ :

$$
\begin{aligned}
& \frac{\partial}{\partial x_{i_{1}}} \mathscr{E}_{[i]}^{(k)}(x) k_{1} \sqrt{c_{[i]}^{(k)}} x_{i_{1}}^{k_{1}-1} \cdots x_{i_{n}}^{k_{n}} \\
& =k_{1} \frac{\sqrt{c_{[i]}^{(k)}}}{\sqrt{c_{[i]}^{\left(k^{1^{\prime}}\right)}}} \cdot \sqrt{c_{[i]}^{\left(k^{1^{\prime}}\right)}} x_{i_{1}}^{k_{1}-1} \cdots x_{i_{n}}^{k_{n}} \\
& =k_{1} \frac{\sqrt{c_{[i]}^{(k)}}}{\sqrt{c_{[i]}^{\left(k^{1^{\prime}}\right)}}} \mathscr{E}_{[i]}^{\left(k^{1^{\prime}}\right)}(x),
\end{aligned}
$$

where $\left(k^{1^{\prime}}\right)=\left(k_{1}-1, k_{2}, \ldots, k_{n}\right)$.

We denote $\gamma_{k_{j}}=k_{j} \sqrt{c_{[i]}^{(k)}} / \sqrt{c_{[i]}^{\left(k^{j^{\prime}}\right)}},\left(k^{j^{\prime}}\right)=\left(k_{1}, k_{2}, \ldots, k_{j}-\right.$ $\left.1, \ldots, k_{n}\right),(j=1, \ldots, n)$.

Let $R=\sup \left\{\gamma_{k_{j}}: k_{j} \geq 1\right\}$. If $D_{a}$ is bounded, then for each positive integer $k_{j}$

$$
\gamma_{k_{j}}=\left\|\frac{\partial}{\partial x_{i_{j}}} \mathscr{E}_{[i]}^{(k)}(x)\right\|_{\eta} \leq\left\|D_{a}\right\| \cdot\left\|\mathscr{E}_{[i]}^{(k)}\right\|_{\eta}=\left\|D_{a}\right\| .
$$

So $R<\infty$.

Conversely, if $R<\infty$, then for every holomorphic polynomial $f$, we have

$$
\begin{aligned}
D_{a} f & =\sum_{t, k_{j}} \sum_{(k),[i]} a_{t} \cdot \gamma_{k_{j}}\left\langle f \mid \mathscr{E}_{[i]}^{(k)}\right\rangle_{\eta} \mathscr{E}_{[i]}^{\left(k^{j^{\prime}}\right)}, \\
\left\|D_{a} f\right\|^{2} & =\sum_{t, k_{j}} \sum_{(k),[i]} a_{t}^{2} \cdot \gamma_{k_{j}}^{2}\left|\left\langle f \mid \mathscr{E}_{[i]}^{(k)}\right\rangle_{\eta}\right|^{2} \leq R^{2}\|f\|^{2} .
\end{aligned}
$$

Since the polynomials are dense in $\mathscr{H}_{\eta}$, it follows immediately that $D_{a}$ is bounded on $\mathscr{H}_{\eta}$, with norm $\leq R$.

For a given $a \in E$ let an operator $T_{a}: \mathscr{H}_{\eta} \rightarrow \mathscr{H}_{\eta}$ be defined as

$$
T_{a}(f)(x)=f(x+a)=\sum_{n=0}^{\infty} \frac{1}{n !} D_{a}^{n} f(x),
$$

where $D_{a}^{n} f(x)$ is the $n$ th-Fréchet derivative of $f$ at the point $x \in E$ towards $a$.

Corollary 16. Suppose the set $\left\{k_{j} \sqrt{c_{[i]}^{(k)}} / \sqrt{c_{[i]}^{\left(k^{j^{\prime}}\right)}}\right\}$ is bounded. Then each translation operator $T_{a}$ is bounded on $\mathscr{H}_{\eta}$, and

$$
T_{a}=\sum_{n=0}^{\infty} \frac{1}{n !} D_{a}^{n}
$$

where the series on the right converges in the norm operator topology.
Proof. It is well known, and not difficult to show, that $T_{a}=$ $\sum_{n=0}^{\infty}(1 / n !) D_{a}^{n}$ holds for the full space $\mathscr{H}_{\eta}$ of entire functions, in the sense that when each term of the series on the right is applied to a function $f \in \mathscr{H}_{\eta}$, the result converges uniformly on bounded subsets of $E$ to the function $f(x+a)$ (see, e.g., [7]).

Once we know this, it only remains to note that since $D_{a}$ is bounded (Theorem 15), the series on the right side of $T_{a}=\sum_{n=0}^{\infty}(1 / n !) D_{a}^{n}$ converges in operator norm to a bounded operator on $\mathscr{H}_{\eta}$, and this bounded operator must be $T_{a}$.

It is relevant to remark that this result can be represented in the form $T_{a}=e^{D_{a}}$ whenever $D_{a}$ is bounded on $\mathscr{H}_{\eta}$.

Theorem 17. Let $E$ be a separable Hilbert space, $x \in E, a \in$ $E, a \neq 0$, and the norm $\|\cdot\|_{\eta}$ is defined on $\mathscr{F}_{\eta}$ such that the differentiation operator $D_{a}$ is continuous, and $\eta$ satisfies condition of Proposition 12 for $g(t)=e^{t}$ and every linear functional $\varphi \in E^{*},\|\varphi\|<\delta$ for some $\delta>0$. That is, $e^{\varphi} \in \mathscr{H}_{\eta}$, $\|\varphi\|<\delta$. Then the operator

$$
\begin{aligned}
T_{a}: \mathscr{H}_{\eta} & \longrightarrow \mathscr{H}_{\eta}, \\
f(x) & \longmapsto f(x+a)
\end{aligned}
$$

is hypercyclic.

Proof. Let $a$ be fixed element from $E$. Consider the function $h: \delta B^{*} \rightarrow \mathbb{C}$ defined by

$$
h(\varphi)=\sum_{n=1}^{\infty} \frac{1}{n !} \varphi^{n}(a),
$$

where $\delta B^{*}=\left\{\varphi \in E^{*}:\|\varphi\|<\delta\right\}$. It is clear that $h: E^{*} \rightarrow \mathbb{C}$ is continuous and nonconstant function. So the sets,

$$
\begin{aligned}
& U:=\left\{\varphi \in \delta B^{*}:\|h(\varphi)\|<1\right\}, \\
& V:=\left\{\varphi \in \delta B^{*}:\|h(\varphi)\|>1\right\},
\end{aligned}
$$

where $\|h(\varphi)\|=\left|\sum_{n=1}^{\infty}(1 / n !) \varphi^{n}(a)\right|$, are both open and nonempty. Hence, according to Lemma 14,

$$
\begin{aligned}
& X_{0}=\operatorname{span}\left\{e^{\varphi}: \varphi \in U\right\}, \\
& Y_{0}=\operatorname{span}\left\{e^{\varphi}: \varphi \in V\right\}
\end{aligned}
$$

are both dense subspaces of $\mathscr{H}_{\eta}$. Next, notice that if $T=T_{a}$, given $\varphi \in \delta B^{*}$,

$$
T\left(e^{\varphi}\right)=\sum_{n=0}^{\infty} \frac{1}{n !} D_{a}^{n}\left(e^{\varphi}\right)=\sum_{n=0}^{\infty} \frac{1}{n !} \varphi^{n}(a) e^{\varphi}=h(\varphi) e^{\varphi} .
$$

By (53),

$$
T^{n} \longrightarrow 0 \text { for } n \longrightarrow \infty \text { pointwise on } X_{0} \text {. }
$$

Also, by Lemma 13 there exists a linear map $S: Y_{0} \rightarrow Y_{0}$ determined by

$$
S\left(e^{\varphi}\right)=[h(\varphi)]^{-1} e^{\varphi}, \quad\left(\varphi \in E^{*}\right)
$$


which by (53) and (56) satisfies

$$
\begin{aligned}
& S^{n} \longrightarrow 0 \text { for } n \longrightarrow \infty \text { pointwise } Y_{0}, \\
& T S=\mathrm{id}_{Y_{0}} \text { on } Y_{0} .
\end{aligned}
$$

By Theorem 1 (Hypercyclicity Criterion), $T=T_{a}$ is hypercyclic.

Note that the translation operator $f(x) \mapsto f(x+a)$ is not hypercyclic in $H^{2}(E)$ in Example 9, because it is discontinuous (see [23]).

Now we consider an example of a special Hilbert space of analytic functions satisfying conditions of Theorem 17.

Example 18. Let us denote by $\mathscr{H}_{\widehat{\eta}}(E)$, where

$$
\widehat{\eta}(x)=\sum_{n=0}^{\infty} \frac{x^{\otimes n}}{(n !)^{2}},
$$

a Hilbert space which consists of bounded-type entire functions on $E$ and

$$
\left\|e_{[i]}^{\otimes(k)}\right\|_{\widehat{\eta}}^{2}=n ! k_{1} ! \cdots k_{n} !
$$

The reproducing kernel of this space is

$$
\begin{aligned}
& k(z, x)=\langle\widehat{\eta}(x) \mid \widehat{\eta}(z)\rangle_{\eta}=\sum_{n=0}^{\infty} \frac{\left\langle x^{\otimes n} \mid z^{\otimes n}\right\rangle_{\eta}}{(n !)^{4}} \\
& =\sum_{n=0}^{\infty} \sum_{(k) \in \mathbb{Z}_{+}^{n}[i] \in \mathbb{N}^{n}} \frac{1}{(n !)^{4}}\left(\frac{n !}{k_{1} ! \cdots k_{n} !}\right)^{2}\left\|e_{i_{1}}^{\otimes k_{1}} \cdots e_{i_{n}}^{\otimes k_{n}}\right\|_{\eta}^{2} x_{i_{1}}^{k_{1}} \\
& \cdots x_{i_{n}}^{k_{n}} z_{i_{1}}^{k_{1}} \cdots z_{i_{n}}^{k_{n}}=\sum_{n=0}^{\infty} \sum_{(k) \in \mathbb{Z}_{+}^{n}} \sum_{[i] \in \mathbb{N}^{n}} \frac{1}{n !} \frac{1}{k_{1} ! \cdots k_{n} !} x_{[i]}^{(k)} z_{[i]}^{(k)} \\
& =\sum_{n=0}^{\infty} \frac{(x \mid z)_{E}^{n}}{(n !)^{2}} .
\end{aligned}
$$

Since the set $\{n(1 / n !) /(1 /(n-1) !)\}=\{1\}$ is bounded, the translation operator $T_{a}$ is bounded and since

$$
\sum_{n=0}^{\infty} \frac{(n !)^{2}\|\varphi\|^{2 n}}{(n !)^{2}}=\sum_{n=0}^{\infty}\|\varphi\|^{2 n}<\infty
$$

for $\|\varphi\|<1, \widehat{\eta}$ satisfies conditions of Proposition 12 and so $T_{a}$ is hypercyclic on $\mathscr{H}_{\widehat{\eta}}(E)$.

\section{Conflict of Interests}

The authors declare that there is no conflict of interests regarding the publication of this paper.

\section{References}

[1] K.-G. Grosse-Erdmann, "Universal families and hypercyclic operators," Bulletin of the American Mathematical Society. New Series, vol. 36, no. 3, pp. 345-381, 1999.
[2] G. D. Birkhoff, "D'émonstration d'un théorème élémentaire sur es fonctions entières," Comptes Rendus de l'Académie des Sciences de Paris, vol. 189, pp. 473-475, 1929.

[3] G. R. MacLane, "Sequences of derivatives and normal families," Journal d'Analyse Mathématique, vol. 2, pp. 72-87, 1952.

[4] F. Bayart and E. Matheron, Dynamics of Linear Operators, vol. 179 of Cambridge Tracts in Mathematics, Cambridge University Press, New York, NY, USA, 2009.

[5] K.-G. Grosse-Erdmann and A. Peris Manguillot, Linear Chaos, Universitext, Springer, London, UK, 2011.

[6] G. Godefroy and J. H. Shapiro, "Operators with dense, invariant, cyclic vector manifolds," Journal of Functional Analysis, vol. 98, no. 2, pp. 229-269, 1991.

[7] R. Aron and J. Bès, "Hypercyclic differentiation operators," Contemporary Mathematics, vol. 232, pp. 39-46, 1999.

[8] Z. Novosad and A. Zagorodnyuk, "Polynomial automorphisms and hypercyclic operators on spaces of analytic functions," Archiv der Mathematik, vol. 89, no. 2, pp. 157-166, 2007.

[9] K. C. Chan and J. H. Shapiro, "The cyclic behavior of translation operators on Hilbert spaces of entire functions," Indiana University Mathematics Journal, vol. 40, no. 4, pp. 1421-1449, 1991.

[10] C. Kitai, Invariant closed sets for linear operators [Ph.D. thesis], University of Toronto, 1982.

[11] R. M. Gethner and J. H. Shapiro, "Universal vectors for operators on spaces of holomorphic functions," Proceedings of the American Mathematical Society, vol. 100, no. 2, pp. 281-288, 1987.

[12] S. Dineen, Complex Analysis on Infinite Dimensional Spaces, Monographs in Mathematics, Springer, New York, NY, USA, 1999.

[13] J. Mujica, Complex Analysis in Banach Spaces, vol. 120 of NorthHolland Mathematics Studies, North-Holland, Amsterdam, The Netherlands, 1986.

[14] O. V. Lopushansky and A. V. Zagorodnyuk, "A Hilbert space of functions of infinitely many variables," Methods of Functional Analysis and Topology, vol. 10, no. 2, pp. 13-20, 2004.

[15] O. Lopushansky and A. Zagorodnyuk, Infinite Dimensional Holomorphy. Spectra and Hilbertian Structures, AGH University of Science and Technology Press, Kraków, Poland, 2013.

[16] S. Saitoh, Integral Transforms, Reproducing Kernels and Their Applications, vol. 369 of Pitman Research Notes in Mathematics Series, Longman, Harlow, UK, 1997.

[17] N. Arcozzi, R. Rochberg, and E. Sawyer, "The diameter spaces, a restriction of the Drury-Arveson-Hardy space," Contemporary Mathematics, vol. 435, pp. 21-42, 2007.

[18] B. J. Cole and T. W. Gamelin, "Representing measures and Hardy spaces for the infinite polydisk algebra," Proceedings of the London Mathematical Society, vol. 53, no. 1, pp. 112-142, 1986.

[19] O. Lopushansky, "Best approximations in Hardy spaces on infinite-dimensional unitary matrix groups," Abstract and Applied Analysis, vol. 2014, Article ID 631503, 8 pages, 2014.

[20] O. Lopushansky and A. Zagorodnyuk, "Representing measures and infinite-dimensional holomorphy," Journal of Mathematical Analysis and Applications, vol. 333, no. 2, pp. 614-625, 2007.

[21] O. Lopushansky and A. Zagorodnyuk, "Hardy type spaces associated with compact unitary groups," Nonlinear Analysis: Theory, Methods \& Applications, vol. 74, no. 2, pp. 556-572, 2011.

[22] K.-H. Neeb and B. Ørsted, "Hardy spaces in an infinite dimensional setting," in Proceedings of the 2nd International Workshop on "Lie Thery and Its Application in Physics", H.-D. Doebrer, V. 
K. Dobrev, and J. Hilgert, Eds., pp. 3-27, Clausthal-Zellerfeld, Germany, 1998.

[23] Z. H. Mozhyrovska and A. V. Zagorodnyuk, "Hypercyclic composition operators on Hilbert spaces of analytic functions," Methods of Functional Analysis and Topology, vol. 20, no. 3, pp. 284-291, 2014. 


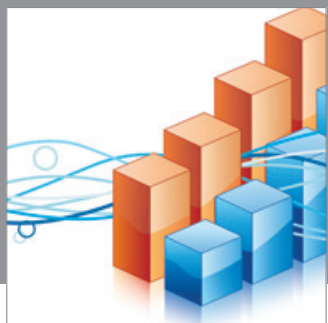

Advances in

Operations Research

mansans

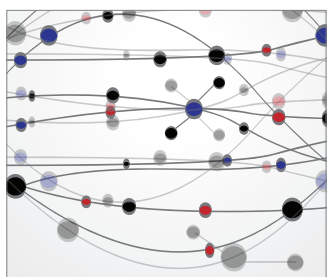

The Scientific World Journal
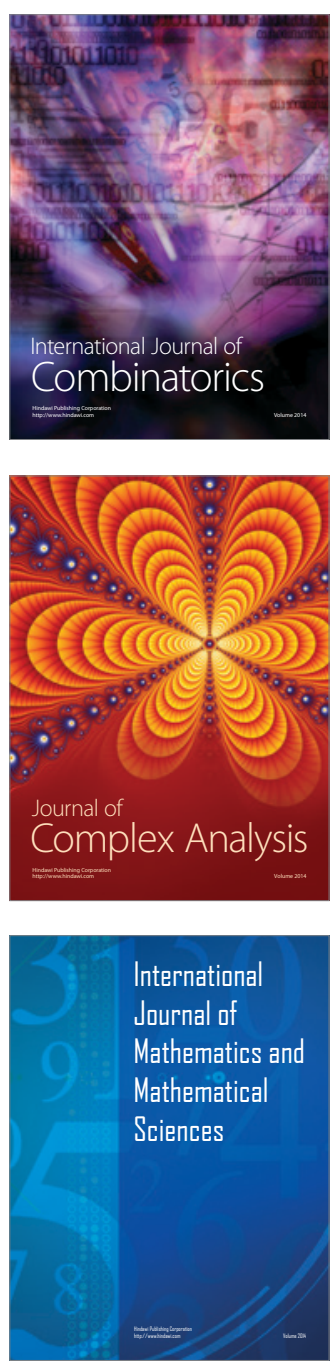
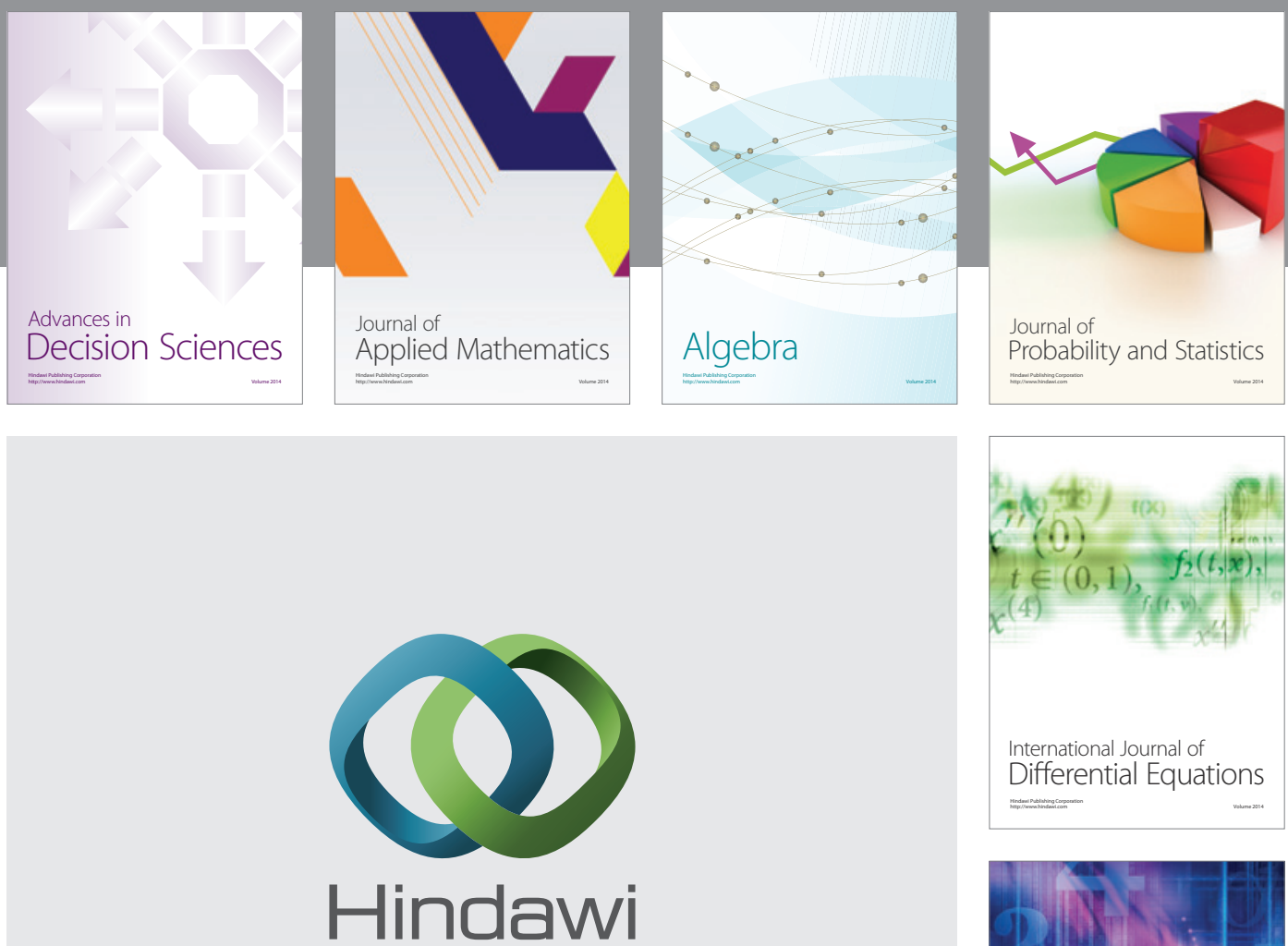

Submit your manuscripts at http://www.hindawi.com
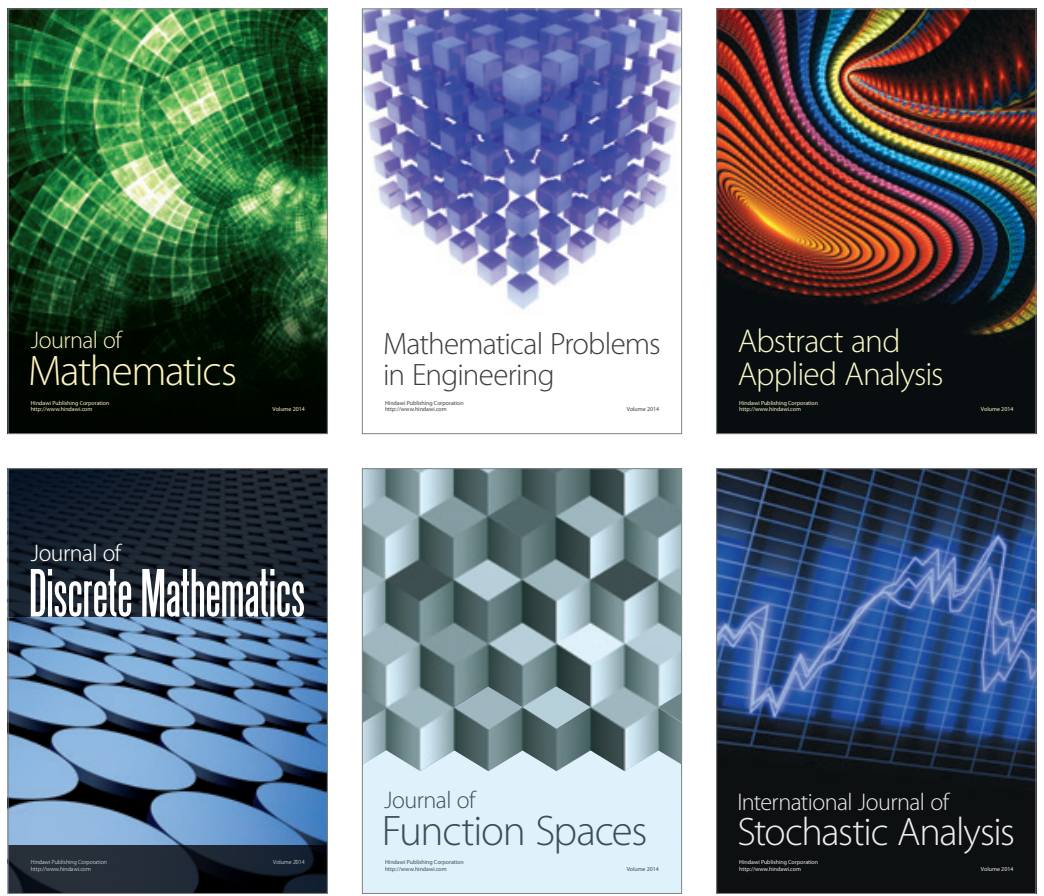

Journal of

Function Spaces

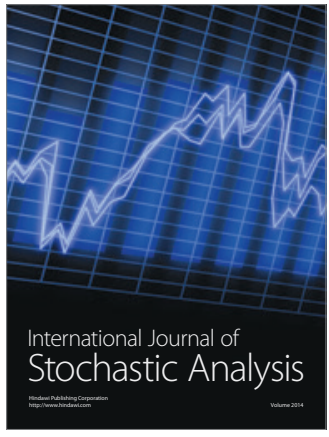

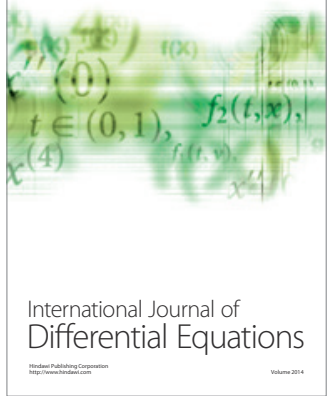
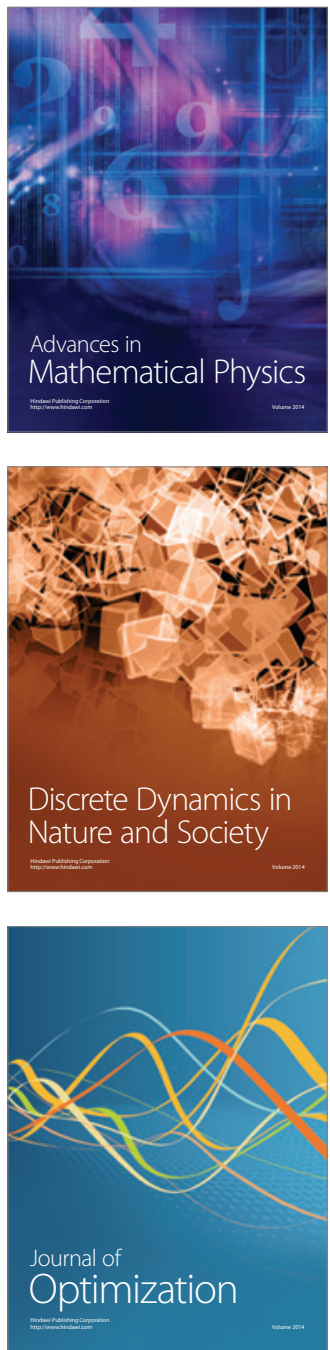